\title{
Using AKF-PSR to Compensate Random Drift Errors of Low-Cost MEMS Gyroscopes
}

\author{
Chunsheng Xiao, Qingyuan Zhu, Huosheng $\mathrm{Hu}^{\circledR}$, Senior Member, IEEE, \\ Xiaoyu Zhang, Xuanwei Chen, and Xinyi Song
}

\begin{abstract}
The random drift of a micro-electromechanical system (MEMS) gyroscope seriously affects its measurement accuracy. To model and compensate its random drift, the time series analysis method has widely been deployed, which, however, requires a large amount of data for pre-processing analysis and is unsuitable for real-time applications. This paper proposes a new random drift compensation method based on the adaptive Kalman filter (AKF) and phase space reconstruction (PSR). $\mathrm{AKF}$ is first designed to compensate the random drift of the low-cost MEMS gyroscope. The phase variables are then used as phase vectors via PSR. Experiments show that the proposed AKF-PSR method can effectively compensate the random drift of the gyroscope, and the standard deviation is reduced by half.
\end{abstract}

Index Terms-MEMS gyroscopes, adaptive Kalman filter (AKF), phase space reconstruction (PSR), random drift errors.

\section{INTRODUCTION}

M EMS gyroscopes are an important part of the inertial navigation system, and commonly used for measuring the angular velocity of a moving object. For instance, MEMS gyroscopes are widely used in the fields of aerospace, autonomous vehicles and robotics due to their small size, low cost and low power consumption. Their measurement accuracy is very much depending on their design and manufacturing processes. In general, the low-cost MEMS gyroscopes may contain random drifts and poor stability, which affect their measurement accuracy and cannot meet the requirements of many real-world applications [1]. Therefore, it is necessary to model and compensate these random drifts.

Recent years, some scholars have used multi-sensor fusion technology to reduce the random drift to improve accuracy

Manuscript received March 9, 2019; revised April 15, 2019; accepted April 16, 2019. Date of publication April 22, 2019; date of current version July 17, 2019. This work was supported in part by the National Natural Science Foundation of China under Grant 51575463, in part by the Fujian Collaboration Innovation Center for R\&D of Coach and Special Vehicle under Grant 2016BJC016, in part by the Key Technology R\&D Program of Fujian under Grant 2016HZ0001-9, and in part the Scholarship under the Education Department of Fujian Province. The associate editor coordinating the review of this paper and approving it for publication was Dr. Stefan J. Rupitsch. (Corresponding author: Qingyuan Zhu.)

C. Xiao, X. Zhang, X. Chen, and X. Song are with the Department of Mechanical and Electrical Engineering, Xiamen University, Xiamen 361005, China.

Q. Zhu is with the Department of Mechanical and Electrical Engineering, Xiamen University, Xiamen 361005, China, and also with the Fujian Collaboration Innovation Centre for R\&D of Coach and Special Vehicle, Xiamen University of Technology, Xiamen 361005, China (e-mail: zhuqy@xmu.edu.cn).

$\mathrm{H}$. Hu is with the School of Computer Science and Electronic Engineering, University of Essex, Colchester CO4 3SQ, U.K.

Digital Object Identifier 10.1109/JSEN.2019.2912400 of MEMS gyroscopes. The main idea is to create a virtual low-drift gyroscope by combining the output of the high-drift gyroscope array [2]. Under the condition that each gyroscope in the sensor array is uncorrelated, it can effectively compensate the random drift of gyroscope and improve the accuracy of the measurement signal [3]-[6]. In order to eliminate the influence of gyroscope correlation, some scholars also consider the use of multiple types of sensors in the gyroscope application to compensate for the random drift. For example, Hong , et al. fused encoder and gyroscope data to obtain heading angle [7], [8]. Sheng, et al. designed a multi-sensor platform to measure angular rate information through complementary fusion, which was based on a 3-axis gyroscope, a 3-axis accelerometer, and a 3-axis magnetic sensor [9].

The application of multi-sensor fusion technology means an increase in cost. In addition, these methods are not applicable when the installation size is limited or in a strong magnetic environment. Therefore, some scholars have proposed to use the drift signal of the gyroscope for self-compensation. Shi. et al. proposed a continuous threshold function based on wavelet threshold de-noising and applied the improved wavelet de-noising algorithm to compensate for gyro drift [10], [11]. Some scholars have also considered using Restricted Boltzmann Machine (RBM) neural networks to construct nonlinear models to predict random drift, and used predictive values to compensate for random drift [12]. Since the neural network has problems such as over-fitting, Xing et al. used statistical methods to design a Least Squares Support Vector Machine (LSSVM) model based on PSR for short-term prediction and compensation of random drift [13].

In addition, Kalman filters have been widely used to process the measurement data in order to obtain accurate compensation in a long term. The key to this approach is to model the random drift signal of the MEMS gyroscope [14], and compensate by establishing a drift model combined with real-time measured signals. Since the random drift of the gyroscope has high random characteristics and it is difficult to describe its state, some researchers use time series models such as MA model [15], AR model [16], ARMA model [17], [18] to model the statistical properties of gyro random drift in the time domain. Considering the nonlinear characteristics of random drift of gyroscopes, some researchers believe that traditional time series linear models cannot accurately describe their characteristics. Therefore, hybrid models such as gray 


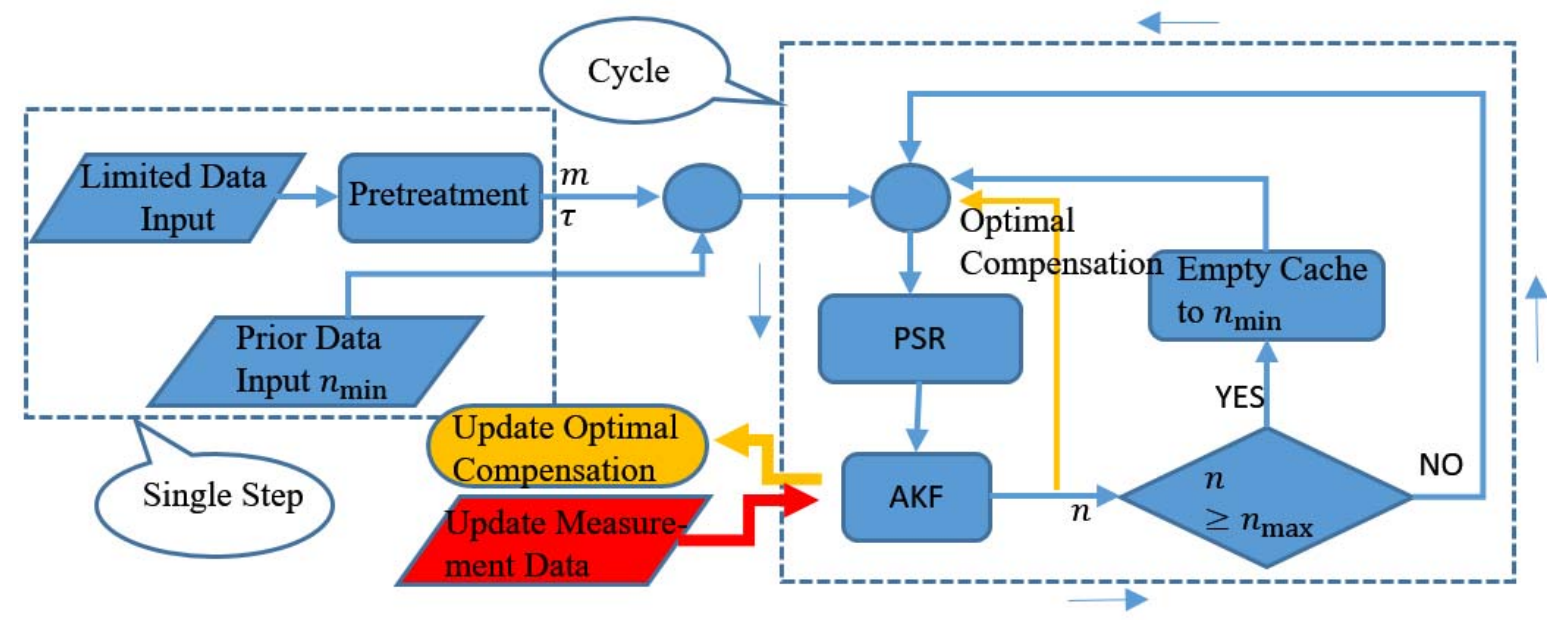

Fig. 1. The flow chart of the proposed AKF-PSR algorithm.

and ARMA model prediction [19], two-stage recursive least squares method [20] were proposed.

The application of time series models (including hybrid models) requires time series normal distribution, zero mean and smooth. It means that using this method to model the random drift of the gyroscope requires extensive pre-processing analysis. In order to avoid a large amount of preprocessing, Kirkko et al. used ten samples of neighbors as state vectors to map the random drift of the gyroscope to high-dimensional space modeling, and proved that this method is feasible through experiments [15]. Sierociuk et al. pointed out that unless the state vector is infinite dimensional, the solution cannot be optimal [21].

Considering that the PSR method can map the time series into a finite-dimensional state space and contains all the characteristics of the original system, we propose an adaptive Kalman filter based on PSR (AKF-PSR) in this paper. The proposed AKF-PSR models the random drift of the gyroscope in a high-dimensional space by citing phase variables. The advantage of this method is that it represents the original state of the system by a state vector of finite dimensions. In addition, this method only needs to retain a certain amount of prior data, and update the a priori data during the iteration process, and does not need to pre-process all the data. At the same time, we conduct practical experimental tests to demonstrate the proposed method is feasible and can effectively compensate the random drift of the gyroscope.

The rest of this paper is organized as follows. Section 2 introduces the principle and implementation of Kalman filtering algorithm and explains phase space reconstruction techniques being deployed. The actual experimental results are presented in Section III to demonstrate the feasibility and good performance of the proposed approach. Finally, a brief conclusion and future work are given in Section IV.

\section{Algorithm Principle}

Kalman filter uses the state equation of a linear system to estimate the data through the input and output of the system, which is difficult to systematically model the random drift of MEMS gyroscope. Therefore, we use the reconstructed phase variable as the state vector to establish a state space model based on the phase variable.

Figure 1 shows the flow chart of the proposed algorithm. As the random drift data of a MEMS gyroscope is noisy, a single-step prior processing is required to obtain a suitable embedding dimension $m$ and lag index $\tau$. The pre-measured data $\left(n_{\min }\right)$ is input into the filter for optimal compensation of each updated measurement data. At the same time, the compensation value is stored as a priori data for the next compensation. The maximum value of the stored data $\left(n_{\max }\right)$ is determined by the system requirements and comprehensive consideration on other factors such as compensation accuracy, computational efficiency, and computer hardware.

\section{A. Priori Processing of Single-Step}

Single-step prior processing is to determine the optimal embedding dimension $m$ and lag index $\tau$ of the MEMS gyroscope. Packard et al. indicated that the phase space of a system can be reconstructed from the time series of a variable, because the time series itself contains information about all the variables involved in the dynamic system [22].

According to the embedded theorem proposed by Smale [23], an m-dimensional embedded phase space can always be found in the sense of topological invariance for the scalar time series of infinitely long and noiseless d-dimensional chaotic attractors. When uniform time series observations are $x_{i}, i=1,2,3 \ldots, N$ and appropriate lag index $\tau$, the phase space is reconstructed for this time series as follows:

$$
X=\left[\begin{array}{clcc}
x_{1} & x_{1+\tau} & \ldots & x_{1+(m-1) \tau} \\
x_{2} & x_{2+\tau} & & x_{2+(m-1) \tau} \\
& \vdots & \ddots & \vdots \\
x_{n-(m-1) \tau} & x_{n-(m-2) \tau} & \cdots & x_{n}
\end{array}\right]
$$

where

$$
m \geq 2 d+1
$$


The key of re-constructing the phase space is to find the appropriate $m$ and $\tau$. The $m$ in the formula (2) characterizes the spatial spacing and geometric invariants of the attractor and $\tau$ reflects the degree of correlation of any two adjacent delay coordinate points. For an ideal infinitely long and noise-free time series, they can be taken as arbitrary values.

However, the random drift of a MEMS gyroscope is noisy, and it is necessary to preferentially remove the coarse error during the normalization process. We use the $3-\sigma$ criteria to select the coarse error value, that is, when the residual error $\Delta v$ of the measured value $x_{i}$ satisfies the formula (3) below, it is considered that $x_{i}$ is a value containing a coarse error.

$$
\begin{aligned}
|\Delta v| & =\left|x_{i}-\bar{x}\right|>3 \sigma \\
\bar{x} & =\frac{1}{N} \sum_{i=1}^{N} x_{i} \\
\sigma & =\sqrt{\frac{1}{N-1} \sum_{i=1}^{N}\left(x_{i}-\bar{x}\right)^{2}}
\end{aligned}
$$

where $\bar{x}$ represents the mean of the input data, $\sigma$ represents the standard deviation, and $N$ is the length of the input data.

To ensure the sampling frequency consistency of the input data, the following processing is performed on the data with the coarse error value.

$$
\begin{cases}x_{i}=\bar{x}+|3 \sigma|, & x_{i}-\bar{x}>|3 \sigma| \\ x_{i}=\bar{x}-|3 \sigma|, & x_{i}-\bar{x}<-|3 \sigma|\end{cases}
$$

In order to avoid the problem of maxima or minima, the data with the coarse error removed is normalized. This means that all $x_{i}$ are placed in the interval $[-1,+1]$ as shown below.

$$
x_{i}=2 * \frac{x_{i}-x_{\min }}{x_{\max }-x_{\min }}-1
$$

After that, we use the $\mathrm{C}-\mathrm{C}$ method proposed by Kim et al. [24] to determine $m$ and $\tau$ by introducing the embedded window width $\tau_{w}$. The calculation is described as follows:

1) The Relevant Integral Function is Introduced Below:

$$
C(m, N, r, \tau)=\frac{2}{M(M-1)} \sum_{1 \leq i<j \leq M} \theta\left(r-\left\|X_{i}-X_{j}\right\|_{+\infty}\right)
$$

where $\mathrm{X}_{i}=\left(\begin{array}{llll}x_{i} & x_{i+\tau} & \cdots & x_{i+(m-1) \tau}\end{array}\right)$

$$
\begin{aligned}
\theta(x) & = \begin{cases}0 & x<0 \\
1 & x \geq 0\end{cases} \\
M & =N-(m-1) \tau
\end{aligned}
$$

where $m$ is the embedded dimension, $N$ is the length of the time series, $r$ is the size of the domain radius, $\tau$ is the lag index, and $M$ is the number of embedded points in the embedded space.

2) Divide the Time Series Into $\tau$ Disjoint Time Series, and Calculate the Statistics as Follows:

$$
\begin{aligned}
S(m, N, r, \tau)=\frac{1}{\tau} \sum_{s=1}^{\tau}\left[C_{s}(\right. & m, N / s, r, \tau) \\
& \left.-C_{s}^{m}(1, N / t, r, \tau)\right]
\end{aligned}
$$

where $m=2,3, \ldots, C_{s}$ represents the relevant integral of the s subsequence.
According to the research work conducted by Durlauf [25], the constraints are as follows.

$$
\left\{\begin{array}{l}
N \geq 500 \\
2 \leq m \leq 5 \\
0.5 \sigma \leq r \leq 2 \sigma
\end{array}\right.
$$

Assume that $r_{1}=0.5 \sigma, r_{2}=1.0 \sigma, r_{3}=1.5 \sigma, \quad r_{4}=2.0 \sigma$, we calculate the mean given by (8) as follows.

$$
\begin{aligned}
\bar{S}(\tau) & =\frac{1}{16} \sum_{m=2}^{5} \sum_{j=1}^{4} S\left(m, N, r_{j}, \tau\right) \\
\Delta \bar{S}(\tau) & =\frac{1}{4} \sum_{m=2}^{5} \Delta S(m, \tau)
\end{aligned}
$$

where

$$
\Delta S(m, \tau)=\max \left\{S\left(m, N, r_{j}, \tau\right)\right\}-\min \left\{S\left(m, N, r_{j}, \tau\right)\right\}
$$

The first local zero of $\bar{S}(\tau)$, or the first local minimum of $\Delta \bar{S}(\tau)$ is taken as the lag index $\tau$.

\section{3) Definition:}

$$
\mathrm{S}_{c o r}(\tau)=|\bar{S}(\tau)|+\Delta \bar{S}(\tau)
$$

The minimum value of $\mathrm{S}_{c o r}(\tau)$ is the first overall maximum time window $\tau_{w}$ corresponding to the time series.

We have

$$
\tau_{w}=(m-1) \tau
$$

where $\tau$ takes the lag index obtained by (13), and the optimal embedding dimension $m$ can be obtained by (14).

\section{B. AKF-PSR Design}

AKF-PSR requires pre-input initial values, including minimum prior data $n_{\min }$, optimal embedding dimension $m$, optimal lag index $\tau$, process noise $W$, process noise covariance $Q$, measurement noise $V$ and measurement noise covariance $R$.

Figure 2 shows the design flow of AKF-PSR. After calculating the corresponding reconstruction dimension $m$ and lag index $\tau$, the phase space reconstruction for a given data set is as shown in equation (1). The last item of $X$ is the last item $x_{n}$ of the given data set. Therefore, when $k=n-(m-1) \tau$, we define state vector below

$$
X(k \mid k)=\left[\begin{array}{llll}
x_{k} & x_{k+\tau} & \cdots & x_{k+(m-1) \tau}
\end{array}\right]
$$

In the phase space, we take out phase points $X_{t j}$, $j=1,2 \ldots l$, which have the closest distance from $X(k)$. In case the system is certain, the value of $X_{t j+1}$ can be used to predict the value of $X(k+1)$ because $X(k+1)$ is also close to $X_{t j+1}$ when $X(k)$ is close to $X_{t j}$.

When $\Delta X$ is the smallest, $X_{t j}$ is what we need.

$$
\Delta X=\left\|X_{t j}-\mathrm{X}_{k}\right\|_{2}
$$

Usually, $l>m+1$. Then, the average value of $X_{t j}$ is $\overline{X_{t j}}$.

$$
\bar{X}_{t j}=\frac{1}{l} \sum_{j=1}^{l} X_{t j}
$$




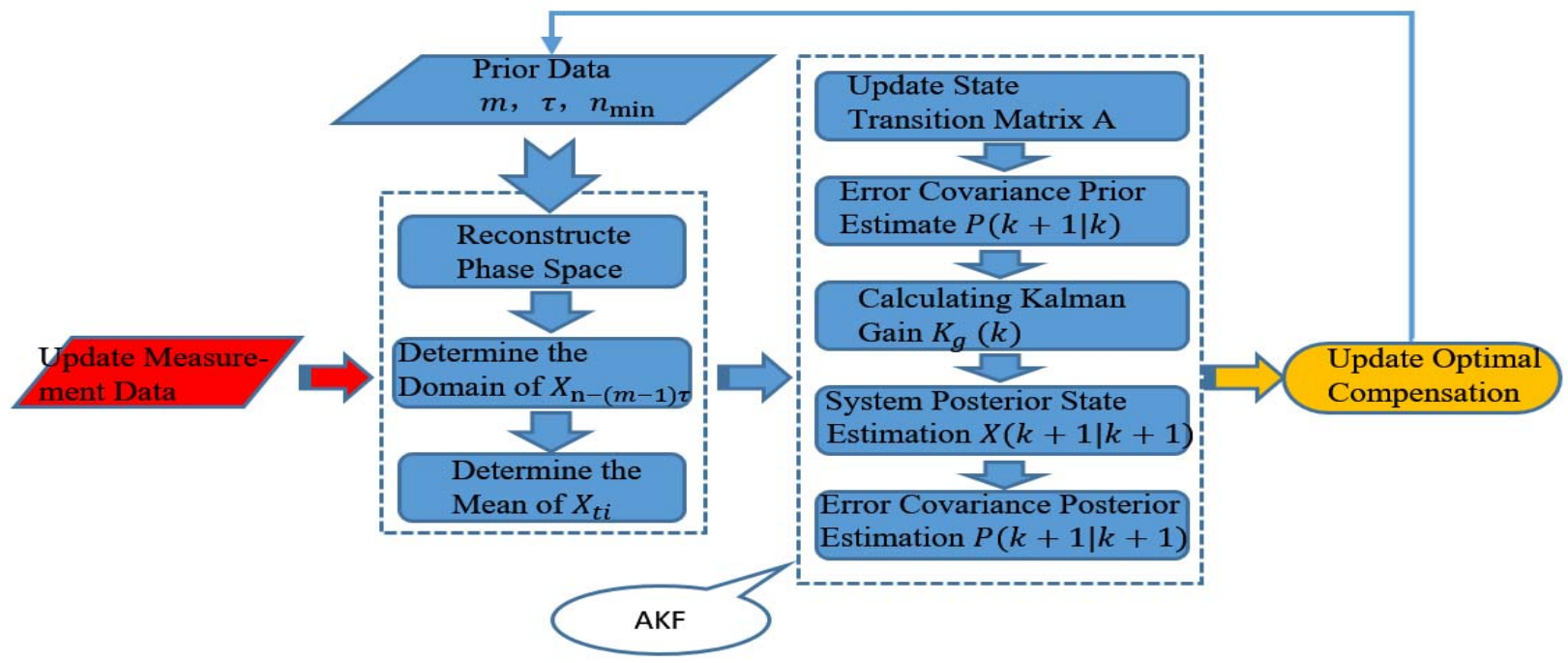

Fig. 2. AKF-PSR design flow of AKF-PSR.

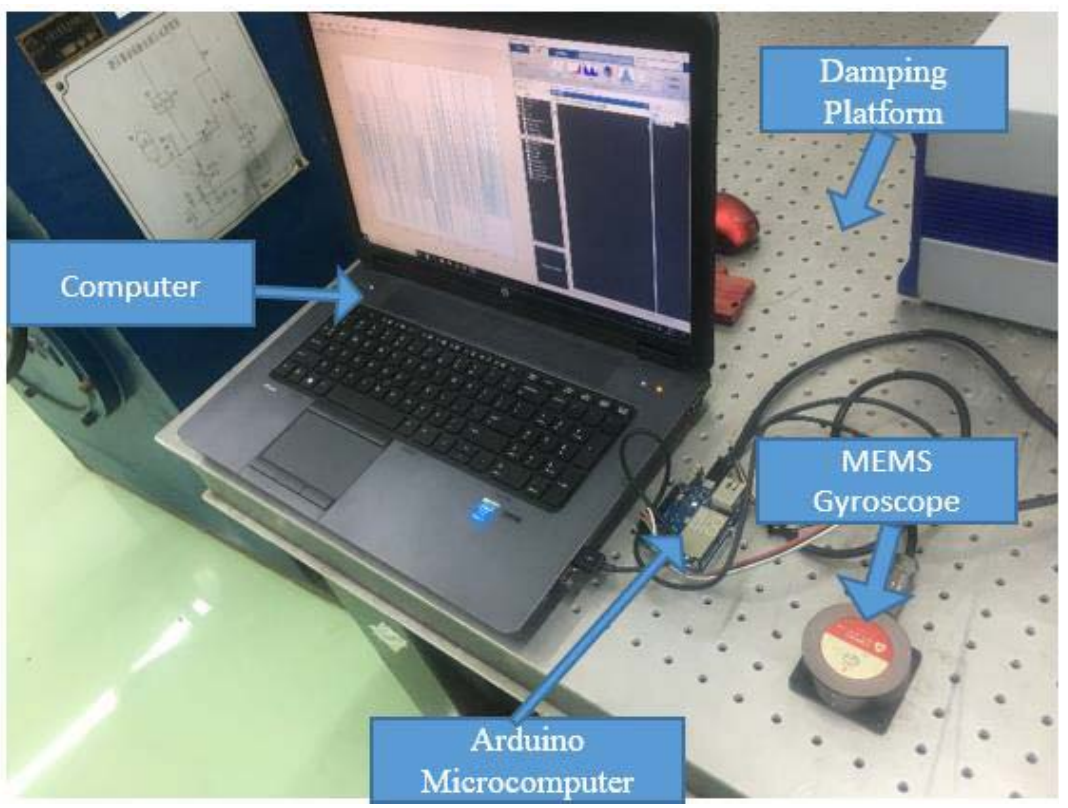

Fig. 3. Experimental environment Setup.

Then we can determine the state transition matrix $A$.

$$
A=X_{k}\left({\overline{X_{t j}}}^{-1}\right)
$$

where ${\overline{X_{t j}}}^{-1}$ represents the inverse of the matrix.

The iteration of Kalman filter can be described below:

1) Estimate the a priori state of the system.

$$
X(k+1 \mid k)=A \overline{X_{t j+1}}+W
$$

where $W$ is the process noise.

2) Calculate error covariance prior estimate.

$$
P(k+1 \mid k)=A P(k \mid k) A^{T}+Q
$$

where $A^{T}$ indicates the transpose of the matrix and $Q$ is the process noise covariance.
3) Calculate Kalman gain $K_{g}(k)$.

$$
K_{g}(k)=P(k+1 \mid k) H^{T} /\left\{H P(k+1 \mid k) H^{T}+R\right\}
$$

where $R$ is the measurement noise covariance.

4) Estimate the posterior state of the system.

$$
\begin{gathered}
X(k+1 \mid k+1)=X(k+1 \mid k)+K_{g}(k)[Z(k+1) \\
-H X(k+1 \mid k)]
\end{gathered}
$$

where

$$
Z(k+1)=H X(k+1)+V
$$

where $\mathrm{H}=1$, and that is, $Z(k+1)$ is the phase variable consisting of measured value at time $k+1 . V$ is the measurement noise 

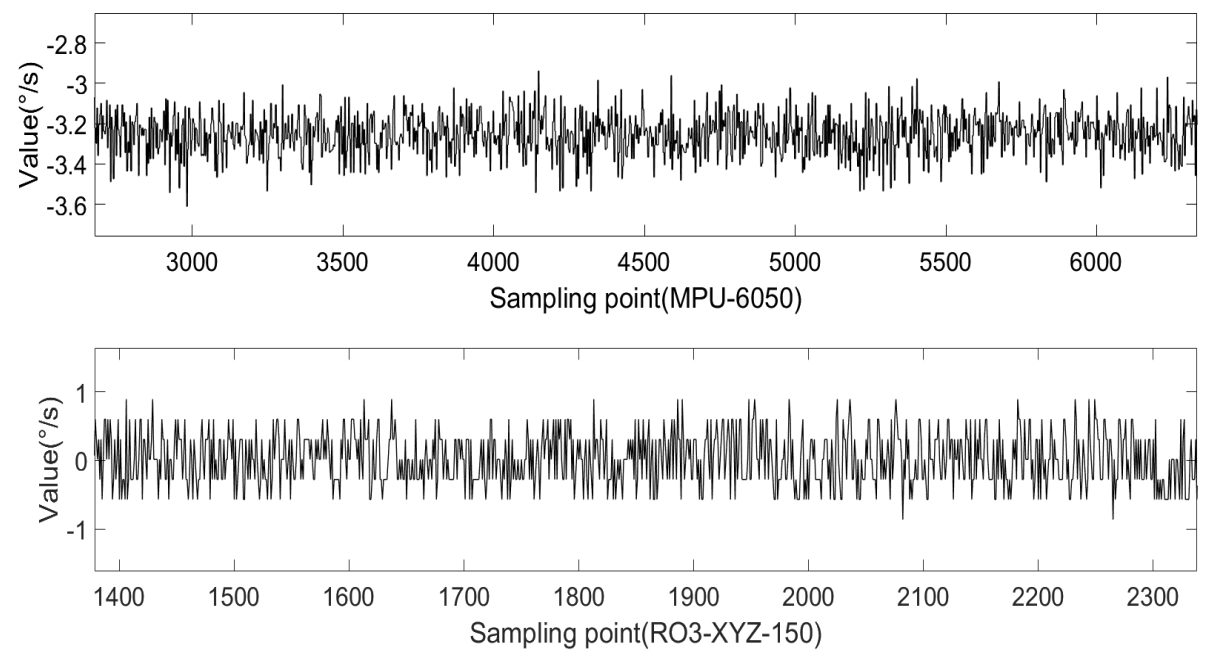

Fig. 4. The X-axis output data of MPU-6050 and RO3-XYZ-150.

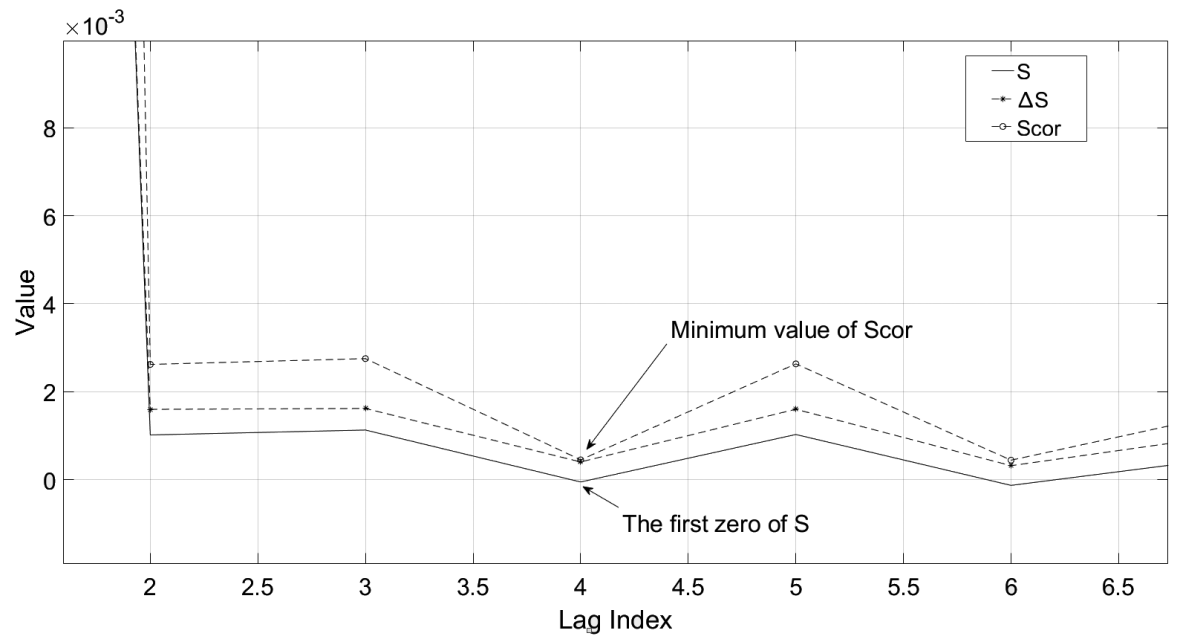

(a)

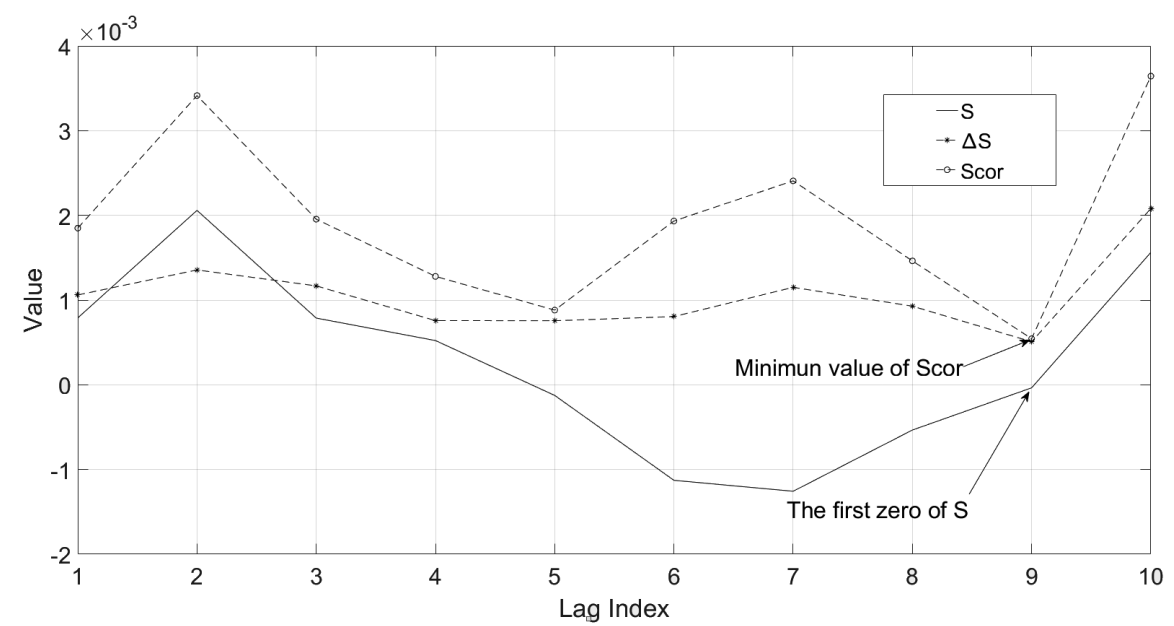

(b)

Fig. 5. (a) The value of $\Delta \bar{S}(\tau), \bar{S}(\tau), \mathrm{S}_{c o r}(\tau)$ vary with the lag index $\tau$ (MPU-6050). (b) The value of $\Delta \bar{S}(\tau), \bar{S}(\tau), \mathrm{S}_{c o r}(\tau)$ vary with the lag index $\tau$ (RO3-XYZ-150).

5) Error covariance posterior estimation.

$$
P(k+1 \mid k+1)=\left[1-K_{g}(k) H\right] P(k+1 \mid k)
$$

After this step, we store the optimally compensated data and use it as the a priori value of the next iteration, i.e. iteration recursively from 1) again. 


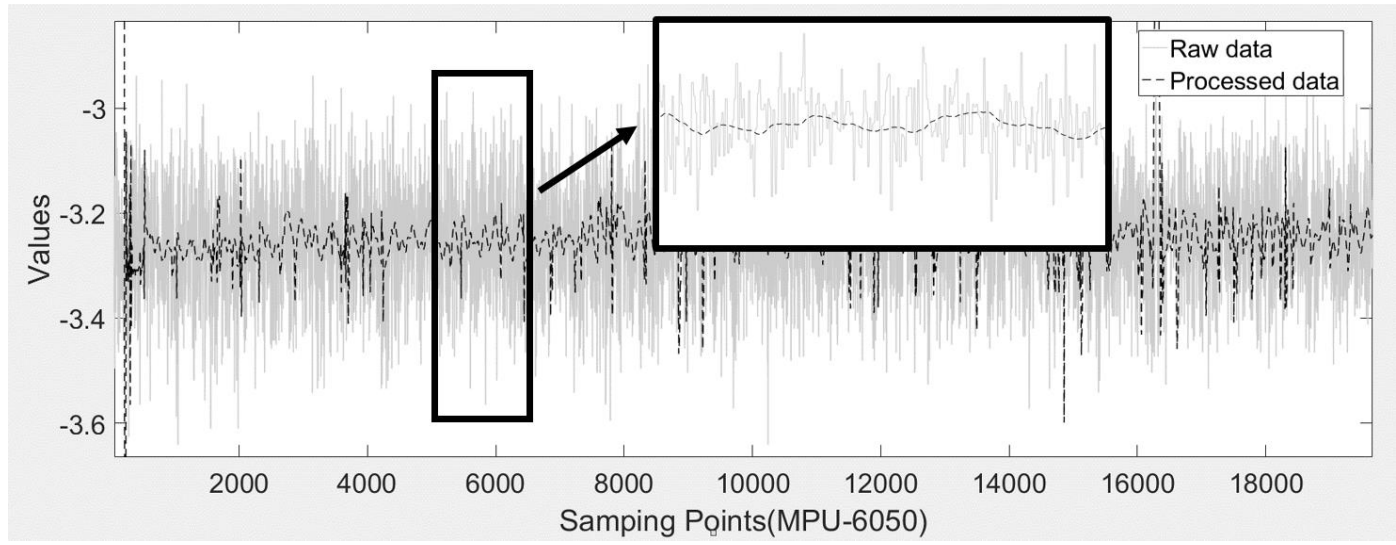

(a)

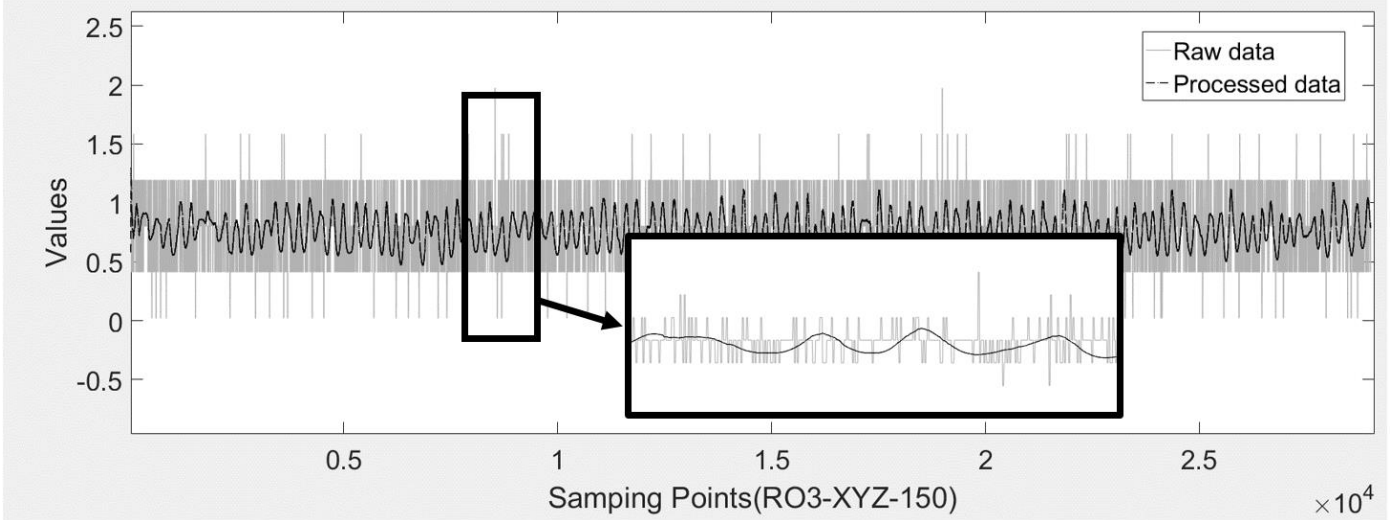

(b)

Fig. 6. (a) The processing results of PSR-AKF (MPU-6050). (b) The processing results of PSR-AKF (RO3-XYZ-150).

\section{TEST AND DISCUSSION}

\section{A. Experiment Setup}

As shown in Figure 3, the gyroscope is attached to the damper platform to reduce noise signals from external sources. Since the MEMS gyroscope's random drift will generally remain stable after it starts up and enters normal operation. After turning on the power, the gyroscope is stationary until the data is stable. In general, the required stabilization time is greater than or equal to the startup time. An Arduino microcomputer is used for the data acquisition, and its sampling frequency is set at $100 \mathrm{HZ}$, which is used to acquire the output angular velocity signal of the X-axis of the MEMS gyroscope and send it to computer for storage analysis and filtering.

In the static case, we collected the $\mathrm{x}$-axis output data of MPU-6050 and RO3-XYZ-150, as shown in Figure 4. Before filtering, the standard deviations of the two gyroscopes are $0.2731^{\circ} / \mathrm{s}$ and $0.2507^{\circ} / \mathrm{s}$ respectively. It should be noted that their built-in temperature sensor compensates for the effects of temperature changes, which ensures random drift to a single variable.

\section{B. Result and Discussion}

1) Comparison of PSR-AKF results on different gyroscopes: In order to verify the applicability of the proposed method, the prior data needs to satisfy $N \geq 500$ according to the constraint conditions proposed by equation (12). When $\mathrm{N}$ tends to infinity, the obtained embedding dimension and lag index are optimal. Considering the limitations of the hardware conditions, we take out the first 50 seconds of the data points (5000 points) of the collected data and calculate the mean $\bar{S}(\tau)$ of the statistic $S$ and its maximum change value $\Delta \bar{S}(\tau)$ by the method described in Section 2.1. Their values vary with the lag index as shown in Figure 5. The minimum value of $\mathrm{S}_{\text {cor }}(\tau)$ corresponds to the first overall maximum time window independent of the time series, taking the first local minimum of $\Delta \bar{S}(\tau)$ or $\bar{S}(\tau)$ as the optimal lag index.

It can be seen from Figure 5(a) that the value of $\bar{S}(\tau)$ is the first zero when $\tau=4$, which is the first optimal lag index. When $\tau$ is $4, \mathrm{~S}_{\text {cor }}(\tau)$ takes the minimum value as the maximum time window $\tau_{w}$. Therefore, the reconstructed phase space of MPU-6050 can be obtained as follows.

$$
X_{M P U-6050}=\left[\begin{array}{cc}
x_{1} & x_{5} \\
x_{2} & x_{6} \\
\vdots & \vdots \\
x_{4996} & x_{5000}
\end{array}\right]
$$

Similarly, the reconstructed phase space of RO3-XYZ-150 can be obtained from Figure 5(b) as follows.

$$
X_{R O 3-X Y Z-150}=\left[\begin{array}{cc}
x_{1} & x_{10} \\
x_{2} & x_{11} \\
\vdots & \vdots \\
x_{4991} & x_{5000}
\end{array}\right]
$$




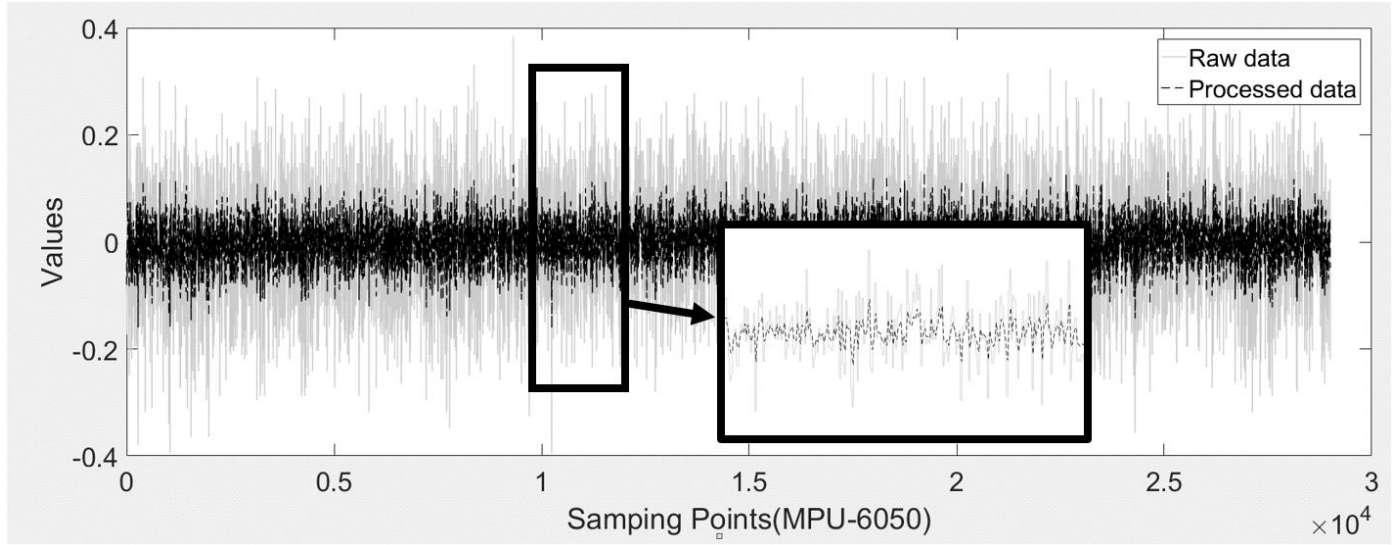

(a)

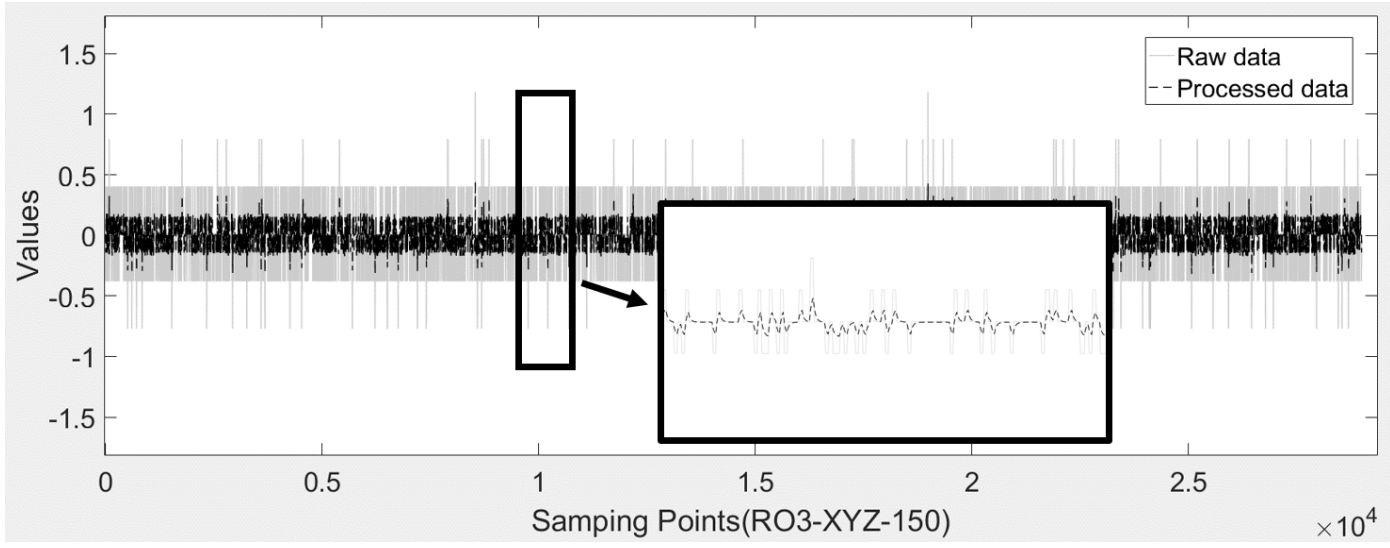

(b)

Fig. 7. (a) The processing results of AR (1) (MPU-6050). (b) The processing results of AR (1) (RO3-XYZ-150).

According to the theory in Section 2.2, we collected 5,000 data points as a priori data of the two gyroscopes, and processed data after that. And then we set the AKF to test the feasibility of the method based on the reconstructed phase space, as shown in Figure 6, in which $R$ and $Q$ are set to the variance of the sample and the residual sequence variance of the model. It can be seen from the filtering results that the proposed method has a good filtering effect on different types of gyroscopes. The standard deviation of MPU-6050 and RO3$\mathrm{XYZ}-150$ is reduced to $0.1041^{\circ} / \mathrm{s}$ and $0.1418^{\circ} / \mathrm{s}$ respectively.

Furthermore, it is apparent from Figure 6 that the filtered values exhibit periodic changes. This means that the prior data exhibits periodic characteristics at a high dimensional level. This also shows that when the a priori data can satisfy the minimum period of high-dimensional space, it can meet the requirements of the algorithm. Ideally, the amount of a priori data $\left(n_{\min }\right)$ should be as small as possible to improve the computational efficiency of the algorithm.

2) Comparison of Proposed Model and AR Model: The AR (MA, ARMA) model is a model that is commonly used to describe time series. The AR (1) model is constructed here to be compared to the method presented in this paper (the modeling process is not specifically described here). Before the AR model is created, all data needs to be preprocessed including removing constant components, removing trend items, and removing periodic items. MPU-6050 (Formula 30) and RO3XYZ-150 (Formula 31) are modeled as follows

$$
\begin{aligned}
& x_{k}=0.6322 x_{k-1}+w_{1} \\
& x_{k}=0.6955 x_{k-1}+w_{2}
\end{aligned}
$$

where $w_{1} \sim \mathrm{N}(0,0.0055), w_{2} \sim N(0,0.0376)$.

Then the Kalman filter is set separately and the results are shown in Figure 7. The standard deviation of MPU-6050 and RO3-XYZ-150 is reduced to $0.0982^{\circ} / \mathrm{s}$ and $0.1651^{\circ} / \mathrm{s}$ respectively. It can be clearly seen that both the proposed method and the AR model can filter the random drift error of the MEMS gyroscope to a certain extent.

Relatively speaking, the AR model is based on the relationship among the current time value, the previous time value and Gaussian white noise. Each iteration is performed by one or several values saved at the previous moment, and it only contains a single linear operation. The method proposed in this paper is to put a priori data $\left(n_{\min }\right)$ into the phase space. When the value of $n_{\min }$ is large, there is no doubt that this processing will greatly occupy the storage space of the computer. In addition, it's needed to make a subtraction for all the data and find the minimum value of group $l$. It is clear that the computational complexity of this approach is much larger than that of the AR model. At the same time, this also shows that we only need to process a certain amount of prior data and save certain data for the next iteration if the 
computer performance is met. This could avoid pre-processing all the data although it reduces the real-time performance of the algorithm.

\section{CONCLUSION}

Modeling and filtering the MEMS gyroscope random drift is an important method to improve its accuracy. In this paper, an adaptive Kalman filter based on phase space reconstruction is proposed to compensate for the random drift of MEMS gyroscopes. The experiment is carried out on different MEMS gyroscopes and compared with the Kalman filter based on AR (1) model to verify the effectiveness and feasibility of the proposed method. The results show that the proposed method can effectively compensate the random drift of the MEMS gyroscope. In the future work, we will consider whether the method works effectively under dynamic conditions and how to improve its computational efficiency.

\section{REFERENCES}

[1] D. Jin and L. Jie, "A compensation algorithnm for zero drifting error of MEMS gyroscope," in Proc. 5th Int. Conf. Meas., Instrum. Automat., in Advances in Intelligent Systems Research, vol. 138, P. Yarlagadda, Ed. Paris, France: Atlantis Press, Jan. 2016, pp. 780-784.

[2] D. S. Bayard and S. R. Ploen, Foundations of Virtual Gyroscope Synthesis, document JPL-D-21656, JPL Internal, Jan. 2002.

[3] H. Chang, L. Xue, C. Jiang, M. Kraft, and W. Yuan, "Combining numerous uncorrelated MEMS gyroscopes for accuracy improvement based on an optimal Kalman filter," IEEE Trans. Instrum. Meas., vol. 61, no. 11, pp. 3084-3093, Nov. 2012.

[4] H. Chang, L. Xue, W. Qin, G. Yuan, and W. Yuan, "An integrated MEMS gyroscope array with higher accuracy output," Sensors, vol. 8, no. 4, pp. 2886-2899, 2008.

[5] X. Ji, "Research on signal processing of MEMS gyro array," Math. Problems Eng., vol. 2015, Jun. 2015, Art. no. 120954.

[6] R. J. Vaccaro and A. S. Zaki, "Reduced-drift virtual gyro from an array of low-cost gyros," Sensors, vol. 17, no. 2, Feb. 2017, p. 352.

[7] S. K. Hong and S. Park, "Minimal-drift heading measurement using a MEMS gyro for indoor mobile robots," Sensors, vol. 8, no. 11, pp. 7287-7299, Nov. 2008.

[8] S. K. Hong, S. C. Lee, J. W. Han, and Y.-S. Ryuh, "Minimaldrift heading measurement using a MEMS gyro for mobile robots: Fused with odometry," Int. J. Control Autom. Syst., vol. 10, no. 5, pp. 1000-1004, Oct. 2012.

[9] H. Sheng and T. Zhang, "MEMS-based low-cost strap-down AHRS research," Measurement, vol. 59, pp. 63-72, Jan. 2015.

[10] X. H. Yang, J. X. Ren, X. M. Zhao, and R. Chen, "MEMS gyro signal de-noising based on adaptive stationary wavelet threshold," in Proc. Intell. Syst. Appl. Mater, in Advanced Materials Research, vols. 466-467, J. H. Wu, M. Zhao, and B. Wu, Eds. Feb. 2012, pp. $986-990$.

[11] Y. S. Shi and Z. F. Gao, "Study on MEMS gyro signal de-noising based on improved wavelet threshold method," in Proc. Adv. Mechatron. Control Eng., in Applied Mechanics and Materials, vols. 433-435, K. Galkowski and Y.-H. Kim, Eds., Oct. 2013, pp. 1558-1562.

[12] J. K. Shiau, D. M. Ma, C. X. Huang, and M. Y. Chang, "MEMS gyroscope null drift and compensation based on neural network," in Proc. Adv. Civil Eng., in Advanced Materials Research, vols. 255-260, J. Zhao, Ed., May 2011, pp. 2077-2081.

[13] H. Xing, B. Hou, Z. Lin, and M. Guo, "Modeling and compensation of random drift of MEMS gyroscopes based on least squares support vector machine optimized by chaotic particle swarm optimization," Sensors, vol. 17, no. 10, Oct. 2017, Art. no. E2335.

[14] S. M. Paniit and W. Zhang, "Modeling random gyro drift rate by data dependent systems," IEEE Trans. Aerosp. Electron. Syst., vol. AES-22, no. 4, pp. 455-460, Jul. 1986.

[15] M. Kirkko-Jaakkola, J. Collin, and J. Takala, "Bias prediction for MEMS gyroscopes," IEEE Sensors J., vol. 12, no. 6, pp. 2157-2163, Jun. 2012.

[16] W. C. Chen, G. W. Gao, J. Wang, L. L. Liu, and X. L. Li, "The study of the MEMS gyro zero drift signal based on the adaptive Kalman filter," in Proc. Adv. Mater. Microwaves Opt., in Key Engineering Materials, vol. 500, D. Wang, Ed., Jan. 2012, pp. 635-639.
[17] W. P. Su, Y. S. Hao, and Q. C. Li, "ARMA-AKF model of MEMS gyro rotation data random drift compensation," in Proc. Mechatron. Ind. Inform., vols. 321-324, P. Yarlagadda and Y.-H. Kim, Eds., Jun. 2013, pp. 549-552.

[18] X.-Y. Jiang, Y.-T. Zong, X. Wang, Z. Chen, and Z.-X. Liu, "Precision improving solutions based on ARMA model and modified self-adapted Kalman filter for MEMS gyro," in Advances Sensor Systems and Applications IV , vol. 7853, B. Culshaw, Y. Liao, A. Wang, X. Bao, $\mathrm{X}$. Fan, and L. Zhang, Eds., (Proceedings of SPIE-The International Society for Optical Engineering), Nov. 2010, Art. no. 78533W.

[19] Z.-J. Zhou and C.-H. Hu, "An effective hybrid approach based on grey and ARMA for forecasting gyro drift," Chaos, Solitons Fractals, vol. 35, no. 3, pp. 525-529, Feb. 2008.

[20] Z. H. Liu, J. B. Chen, Y. L. Mao, and C. L. Song, "MEMS gyro random drift model parameter identification based on two-stage recursive least squares method," in Proc. Adv. Manuf. Technol., vols. 220-223, Z. Jiang, Y. Li, X. Zhang, J. Wang, and W. Sun, Eds., (Applied Mechanics and Materials), Nov. 2012, pp. 1044-1047.

[21] D. Sierociuk, I. Tejado, and B. M. Vinagre, "Improved fractional Kalman filter and its application to estimation over lossy networks," Signal Process., vol. 91, no. 3, pp. 542-552, Mar. 2011.

[22] N. H. Packard, J. P. Crutchfield, J. D. Farmer, and R. S. Shaw, "Geometry from a time series," Phys. Rev. Lett., vol. 45, no. 9, p. 712, Sep. 1980.

[23] S. Smale, "Lecture III dynamical systems and turbulence," in Turbulence Seminar. Berlin, Germany: Springer-Verlag, 1977, pp. 48-70.

[24] H. S. Kim, R. Eykholt, and J. D. Salas, "Nonlinear dynamics, delay times, and embedding windows," Physica D, vol. 127, nos. 1-2, pp. 48-60, Mar. 1999.

[25] W. A. Brock, D. A. Hsieh, and B. LeBaron, Nonlinear Dynamics, Chaos and Instability: Statistical Theory and Economic Evidence. Cambridge, MA, USA: MIT Press, 1991

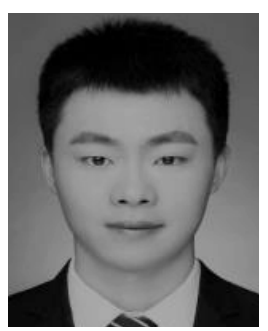

Chunsheng Xiao received the B.Eng. degree from the Department of Mechanical and Electrical Engineering, Xiamen University, China, where he is currently pursuing the degree.

His research interests are vehicle dynamic simulation and modeling.

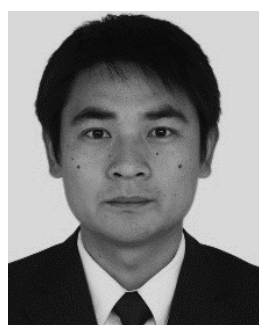

Qingyuan Zhu received the bachelor's and $\mathrm{Ph} . \mathrm{D}$. degrees in vehicle engineering from China Agricultural University, Beijing, China, in 2002 and 2009, respectively.

He was a Visiting Researcher with the School of Engineering, University of Illinois at UrbanaChampaign, Champaign, IL, USA, in 2007. He is currently an Associate Professor with the Department of Mechanical and Electrical Engineering, Xiamen University, Xiamen, China. His current research interests include advanced driver assistance system, sensing and modeling, heavy-duty construction equipment, and off-road vehicles.

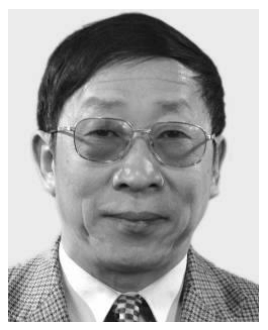

Huosheng $\mathbf{H u}$ is currently a Professor with the School of Computer Science and Electronic Engineering, University of Essex, U.K., where he leads the Robotics Research Group. He has authored over 500 papers. His research interests include robotics, human-robot interaction, mechatronics, embedded systems, and cloud computing.

Prof. Hu is a Fellow of the Institution of Engineering and Technology and the Institute of Measurement and Control. He has been the Program Chair for many IEEE international conferences, such as IEEE ICRA, IROS, ICMA, and ROBIO. He currently serves as the Editorin-Chief for the International Journal of Automation and Computing and Robotics journal (MDPI) and an Executive Editor for the International Journal of Mechatronics and Automation. 


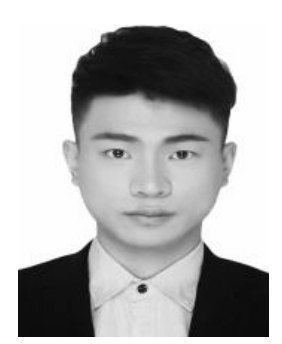

Xiaoyu Zhang received the B.Eng. degree from the School of Logistics and Transportation, Central South University of Forestry and Technology, Changsha, China. He is currently pursuing the degree with the Department of Mechanical and Electrical Engineering, Xiamen University, China.

His research interests are vehicle dynamic simulation and modeling, and control of autonomous vehicles.

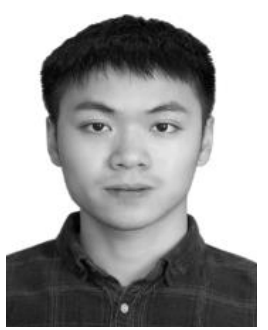

Xuanwei Chen received the B.Eng. degree from the Department of Mechanical and Electrical Engineering, Tan Kah Kee College, Xiamen University, China, where he is currently pursuing the degree.

His research interest is data mining.

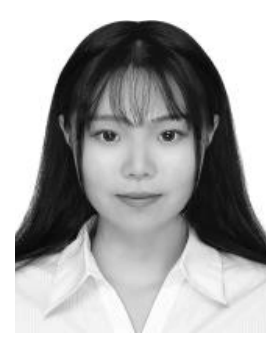

Xinyi Song received the B.Eng. degree from the Department of Vehicle Engineering, Nanjing University of Science and Technology, China. She is currently pursuing the degree with the Department of Mechanical and Electrical Engineering, Xiamen University, China.

Her research interest is the identification of obstacles in driverless cars. 